\title{
On the Measurement of Rotational Spectra of Monodeuterated Asymmetric Top Molecules in Natural Abundance Demonstrated for Monofluorobenzene
}

\author{
H. Dreizler, U. Andresen, J.-U. Grabow, and D. H. Sutter \\ Institut für Physikalische Chemie der Christian-Albrechts-Universität, \\ Olshausenstr. 40, D-24098 Kiel
}

Z. Naturforsch. 53 a, 887-890 (1998); received September 12, 1998

\begin{abstract}
We report on the measurement of rotational transitions of monodeuterated fluorobenzene in natural abundance to show the feasibility of the investigation of monodeuterated asymmetric top molecules without the necessity of isotopic enrichment.
\end{abstract}

\section{Introduction}

A fundamental application of microwave or rotational spectroscopy of molecules is the determination of their structure. Because the analysis of an asymmetric top spectrum provides three rotational constants or moments of inertia, at most three structural parameters can be determined. Therefore the spectra of isotopomers are investigated and used for the structure determination under the assumption that the structure is unchanged upon substitution of isotopes or at least changed only to a small extend. For details see Chapt. XIII of Gordy and Cook [1] and citations therein. One method, the $r_{\mathrm{s}}$ substitution method, introduced by Costain [2] on the basis of equations given by Kraitchman [3], requires in principle the single substitution of each atom in the molecule for the determination of a complete structure from the differences in the moments of inertia. More elaborate structure determinations [4] also rely on the experimental moments of inertia for a sufficient set of isotopomers. A survey of determined structures is given in [5]. For many molecules the preparation of the different isotopomers is a difficult and possibly expensive task. We intend to show that this preparation work can be reduced, since the investigation of many deuterated asymmetric top molecules is becoming feasible in natural abundance.

Over the past years, molecular beam (MB) Fourier transform microwave (FTMW) spectroscopy was in-

Reprint requests to Prof. H. Dreizler; Fax: +49431880 1416, E-mail: dreizler@phc.uni-kiel.de. troduced [6] and considerably improved [7 - 11]. It was noticed that the rotational spectra of many isotopomers can be recorded in natural abundance, assigned and evaluated. This is a consequence of the increased sensitivity compared to MW absorption spectroscopy techniques and the fact, that only lower levels of the rotational energy ladder are populated in a supersonic jet expansion, thus both simplifying the otherwise dense spectra as well as significantely enhancing the remaining transitions. As examples for the observation of rotational spectra of isotopomers containing, in natural abundance, carbon${ }^{13} \mathrm{C}(1.107 \%)$ [12], sulfur ${ }^{33} \mathrm{~S}(0.76 \%)$ [13], nitrogen${ }^{15} \mathrm{~N}(0.3663 \%)$ [14] and other isotopes with higher natural abundance, such as ${ }^{10} \mathrm{~B},{ }^{34} \mathrm{~S}$, may be mentioned. Since many molecules contain hydrogen, $\mathrm{H}$, we tackled the problem to record rotational spectra of fairly heavy asymmetric tops with deuterium, D, in natural abundance $(0.01492 \%)$. Sometimes hydrogen is situated in equivalent positions in the molecule so that the natural abundance increases by the number of equivalent positions. In general, the measurement of deuterated isotopomers of a small linear, prolate symmetric top or even near prolate asymmetric top molecules is an easier task, since the partition sum is rather small in these cases.

\section{Experimental}

We used our MB FTMW spectrometer [11] in a new version and a new arrangement of the microwave circuits [15] with a reduced noise figure. To demonstrate the progress in sensitivity we present in Fig. 1 the 


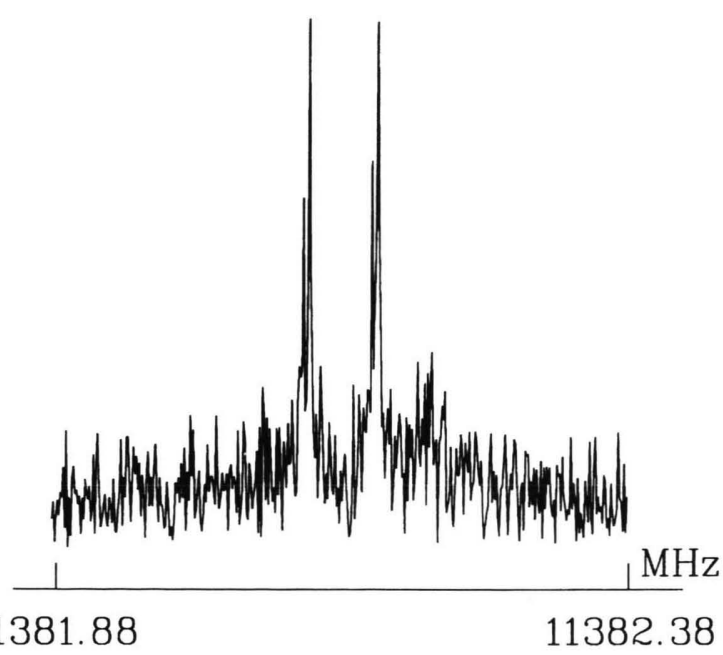

Fig. 1. Fourier transform amplitude spectrum of the $J=1-0$ transition of ${ }^{18} \mathrm{O}^{13} \mathrm{C}^{32} \mathrm{~S}$ in natural abundance $(0.0022 \%)$ of one single transient emission signal. This transition was used to check the spectrometer for sensitivity. The wide doublet results from the Doppler effect due to the parallel arrangement of the molecular beam and the resonator axis [8], while the narrow doublet is due to the ${ }^{13} \mathrm{C}$-spin-rotation coupling ( $\nu=11382.1239 \mathrm{MHz}$ and $\nu=11382.1288$ $\mathrm{MHz}$, resp.). Experimental conditions: one experiment cycle; $10 \mathrm{~ns}$ sampling interval; $8 \mathrm{k}$ data points in the time domain; $4 \mathrm{k}$ spectral points.

Fourier transform amplitude spectrum of carbonylsulfid, ${ }^{18} \mathrm{O}^{13} \mathrm{C}{ }^{32} \mathrm{~S}$, with a natural abundance of $0.0022 \%$ derived from a single transient emission signal (one experiment cycle) with a signal to noise $(S / N)$ ratio of 6. Isotopomers with minor abundance require more experiment cycles.

As asymmetric top test molecule we have chosen monofluorobenzene, $\mathrm{C}_{6} \mathrm{H}_{5} \mathrm{~F}$, as this molecule was investigated earlier with all its isotopomers using isotopically enriched samples $[16,17]$. The deuterium isotopomers have been recently investigated by $\mathrm{MB}$ FTMW spectroscopy [17]. Fluorobenzene was supplied by Aldrich, Steinheim, and was used without further purification. Using the ${ }^{13} \mathrm{C}$ isotopomers [16] we adjusted the experimental parameters (i. e. polarizing power, MW pulse width, molecular pulse width, delay between molecular and MW pulse, delay between MW pulse and data acquisition, beam nozzle operation, and the alignment of the MW antennae) until we were able to record the corresponding transition in one experiment cycle. The sample was prepared in a storage container by mixing $2.5 \%$ of fluorobenzene in neon as carrier gas at $200 \mathrm{kPa}$ total pressure. For spectroscopy, the backing pressure was varied

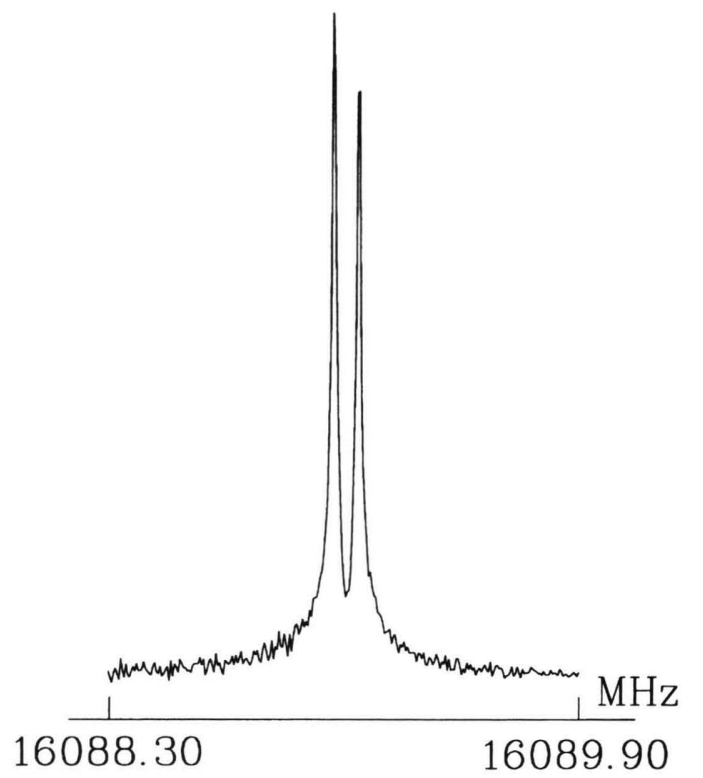

Fig. 2. $1.6 \mathrm{MHz}$ section of the Fourier transform amplitude spectrum of the $J_{K_{a} K_{c}}=4_{04}-3_{03}$ transition of fluorobenzene-2 ${ }^{13} \mathrm{C}$ digitizing one transient emission signal after optimization of the spectrometer. Experimental conditions: $\nu=16089.1043 \mathrm{MHz}$; one experiment cycle; $10 \mathrm{~ns}$ sampling interval; $16 \mathrm{k}$ data points in the time domain; $8 \mathrm{k}$ spectral points.

between 100 and $140 \mathrm{kPa}$. The quality of such an optimized signal is demonstrated for the $J_{K_{a} K_{c}}=4_{04}-3_{03}$ transition of fluorobenzene- $2{ }^{13} \mathrm{C}$, with $2.214 \%$ natural abundance in Figure 2. By reproducing a number of the transitions reported in Table 1 to 3 of [17] of fluorobenzene- $n$ - $\mathrm{D}_{1}, n=2,3,4$, we noticed that transitions with low $K_{a}$-quantum number and unresolvable D-hfs can be recorded with a tolerable number of experiment cycles. The $J_{K_{a} K_{c}}=4_{04}-3_{03}$ transition is an example for those transitions. These transitions for the fluorobenzene- $2 \mathrm{D}_{1}$, and $3 \mathrm{D}_{1}$ were not reported in [17], but the rotational parameters of Table 4 of [17] allowed a good prediction of the frequencies of these transitions. To demonstrate the possibility to locate the transitions with a frequency scan [10], such as is typically used in the process of recording of a yet unassigned spectrum, we also present such a scan form 15886.5 to $15900 \mathrm{MHz}$ for the $2 \mathrm{D}_{1}$ isotopomer and from 15752.3 to $15758.3 \mathrm{MHz}$ for the $3 D_{1}$ isotopomer in the inserts of Figs. 3 and 4 . For these scans $10 \mathrm{~ns}$ sample intervals, $2 \mathrm{~K}$ data points per transient emission, and 512 experiment cycles per polarization frequency setting at a repetition rate of $5 \mathrm{~Hz}$ were used. The polarization frequency stepwidth of 


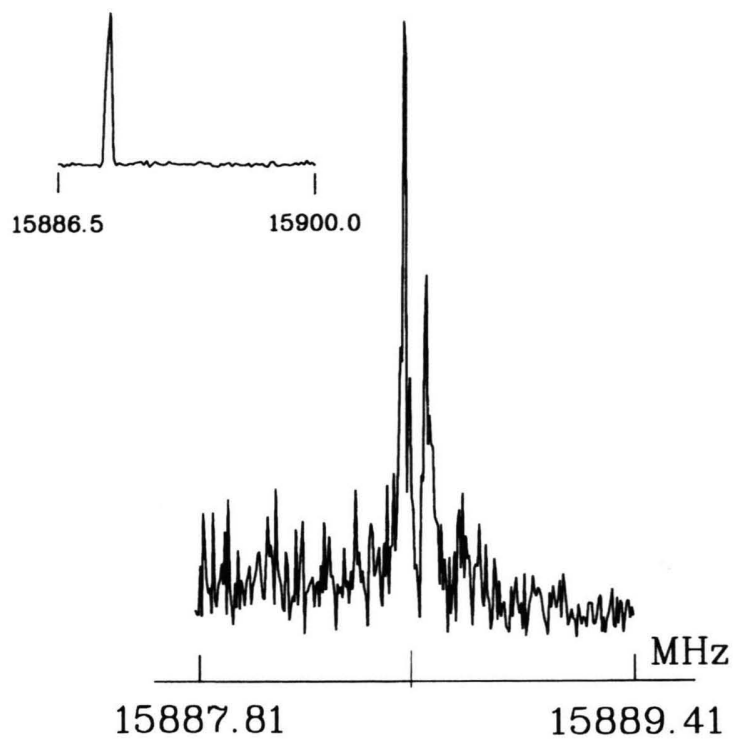

Fig. 3. Upper left: frequency scan from $\nu=15886.5 \mathrm{MHz}$ to $\nu=15900.0 \mathrm{MHz}$ used to locate the fluorobenzene-2D $J_{K_{a} K_{c}}=4_{04}-3_{03}$ transition. Experimental conditions: 512 experiment cycles; $10 \mathrm{~ns}$ sampling interval; $2 \mathrm{k}$ data points; $0.15 \mathrm{MHz}$ frequency step width. Right: $1.6 \mathrm{MHz}$ section of the high resolution spectrum of the same transition. Experimental conditions: $\nu=15886.6169 \mathrm{MHz} ; 1024$ experiment cycles; $10 \mathrm{~ns}$ sampling interval; $32 \mathrm{k}$ data points in the time domain; $16 \mathrm{k}$ spectral points.

the scans was $0.15 \mathrm{MHz}$. In Figs. 3 and 4 we give the subsequently recorded transitions with 10 ns sample interval, $16 \mathrm{~K}$ data points per transient emission signal and 1024 experiment cycles at $15888.617 \mathrm{MHz}$ and $15754.381 \mathrm{MHz}$. These lines fit with a standard deviation of $2 \mathrm{kHz}$ and $6 \mathrm{kHz}$, respectively, to a Watson $\mathrm{A}$ centrifugal distortion analysis [18] using the lines in Tables 2 and 3 of [17]. A similar standard deviation was obtained when only the transitions given in [17] are used. So we are rather sure of the assignment. An additional line at $15888.718 \mathrm{MHz}$ was excluded as the standard error of the fit deteriorated by a factor of 7 . The $J_{K_{a} K_{c}}=4_{04}-3_{03}$ of fluorobenzene- $4 \mathrm{D}_{1}$ given in [17] could be recorded under the same settings with an $S / N$ ratio of about 25 .

\section{Conclusion}

We demonstrated that for a fairly asymmetric top molecule it is possible to record rotational spectra of monodeuterated isotopomers in natural abundance

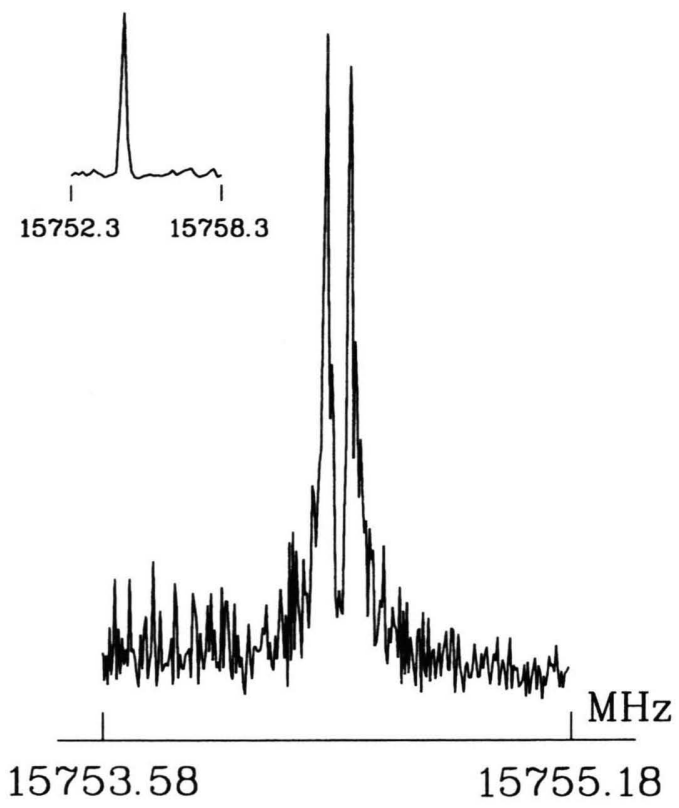

Fig. 4. Upper left: frequency scan from $\nu=15752.3 \mathrm{MHz}$ to $\nu=15758.3 \mathrm{MHz}$ used to locate the fluorobenzene-3D $J_{K_{a} K_{c}}=4_{04}-3_{03}$ transition. Right: $1.6 \mathrm{MHz}$ section of the high resolution spectrum of the same transition. $\nu=$ 15754.3810 MHz. Experimental conditions: see Fig. 3 for both recordings.

using a sensitive and well adjusted MB FTMW spectrometer. To our knowledge, measurements of this kind were not reported hithertoo. Even though a general application is still a challenge, this result is encouraging since such asymmetric top molecules exhibit rather large partition functions compared to near prolate asymmertric top, prolate symmetric top, or even linear molecules. With the improvement of the sensitivity of the MB FTMW spectrometers, a wide field of structure determination and of the analysis of deuterium nuclear quadrupole couplings may be opened without the necessity for possibly difficult and expensive chemical preparations.

\section{Acknowledgement}

We thank the members of our group for help and discussions, and especially the mechanics workshop of our institute for the indispensable help during construction work. The funds were provided by the Deutsche Forschungsgemeinschaft, the Fonds der Chemie and the Land Schleswig-Holstein. 
[1] W. Gordy and R. L. Cook, Microwave Molecular Spectra, J. Wiley, New York 1984.

[2] C. C. Costain, J. Chem. Phys. 29, 864 (1958).

[3] J. Kraitchmann, Amer. J. Phys. 21, 17 (1953).

[4] H.D. Rudolph, Advances in Molecular Structure Research 1, 63 - 114 (1995). J. Demaison, G. Wlodarczak, and H.D. Rudolph, Advances in Molecular Structure Research 3, 1 - 51 (1997).

[5] G. Graner, E. Hirota, T. Iijima, K. Kuchitsu, D. A. Ramsay, and J. Vogt, in Structural Data of Free Polyatomic Molecules, K. Kuchitsu Ed. LandoltBörnstein, Numerical data and functional relationships in science and technology, Springer Berlin 1995, Group II, Vol. 23 and preceeding volumes II/7, II/15, and II/21.

[6] T. J. Balle and W. H. Flygare Rev. Scient. Instrum. 52, 33 (1981).

[7] R.D.Suenram, F. Lovas, G.T. Fraser, J.Z. Gillies, C. W. Gillies, and M. Onda J. Mol. Spectrosc. 137, 127 (1989).

[8] J.-U. Grabow and W. Stahl, Z. Naturforsch., 45a, 1043 (1990).
[9] C. Chuang, C. J. Hawley, T. Emilson, and H.S. Gutowsky, Rev. Scient. Instrum. 61, 1629 (1990).

[10] U. Andresen, H. Dreizler, J.-U. Grabow, and W. Stahl, Rev. Scient. Instrum. 64, 3694 (1990).

[11] J.-U. Grabow, W. Stahl, and H. Dreizler, Rev. Scient. Instrum. 67, 4072 (1996).

[12] D. Consalvo and W. Stahl, J. Mol. Struct. 447, 119 (1998).

[13] B. Kirchner, H. Huber, G. Steinebrunner, H. Dreizler, J.-U. Grabow, and I. Merke, Z. Naturforsch., 52a, 297 (1997) and citations herein.

[14] S. Huber, T.-K. Ha, and A. Bauder, J. Mol. Struct. 413/414, 93 (1997).

[15] J.-U. Grabow et al., to be published.

[16] L. Nygaard, I. Bojesen, T. Petersen, and J. RastrupAndersen, J. Mol. Struct. 2, 209 (1968).

[17] S. Jans-Bürli, M. Oldani, and A. Bauder, Mol. Phys. 68, 1111 (1989).

[18] J. K. G. Watson, in J. R. Durig (Ed), Vibrational Spectra and Structure, Elsevier, Amsterdam 1977, p. 1. 